\title{
Feature extraction of the brain tumours with the help of MRI, based on symmetry and partitioning
}

\author{
Pratima Gumaste ${ }^{1}$, Vinayak Bairagi ${ }^{2}$ \\ ${ }^{12}$ AISSM's, Institute of Information Technology
}

\section{Article Info}

Received Apr 4, 2019

\section{Keyword:}

MRI,

Mid-sagittal plane,

symmetry,

T2 weighted Images

\begin{abstract}
Computer-aided diagnostic (CAD) studies are used for scientific observations for explanation since very long time, but they are extraordinarily powerful to perform completely machine-driven algorithmic analyses for brain magnetic resonance imaging lesions. Structural and purposeful imbalance within the human brain could be reviewed. This imbalance analysis of the brain has terrific importance in an image analysis. In the present work, the imbalance between the two hemispheres is considered as the base for the detection of the tumour. We have segmented the brain into the two halves using thresholding technique, followed by statistical feature extraction for the double authentication of the existence of tumour which proves to be the better approach. The approach also takes into consideration corrections needed for the tilt observed while capturing the MRI.
\end{abstract}

\section{Corresponding Author:}

Pratima Gumaste,

Research Scholar, AISSM's, Institute of Information Technology

Email: p_gumaste@rediffmail.com

\section{Introduction}

In trendy medication practices, medical pictures play a significant role and treated as necessary tools for the patient's designation and treatment. It is terribly troublesome to mechanically find solely the areas of illness in the medical pictures. It is an awfully troublesome method. Thanks to the extraordinarily massive volumes of the knowledge and extreme quality. Computer-aided designation (CAD) is used as an awfully useful gizmo to modify automatic interpretations of the medical pictures. But, it is established to be astonishingly troublesome to perform absolutely machine-driven algorithmic analyze on lesions, exclusively on the data contained in the pictures. Several analysis techniques are evolved for mechanically recognizing the areas of the brain tumor and lump in human brain. To make powerful the processed systems, it might appear insightful to include information and heuristics of disciplines akin to anatomy and pathology to assist the arrangement depict diagnostic inferences.

The CAD system will offer a precious assessment and accuracy of prior tumor recognition. The system consists of 2 stages. The primary stage involves pre-processing and improvement, whereas within the second stage, characteristics pulling out, characteristic choice, categorization, and performance examination are compared and studied. Pre-processing and enhancementmethods are helpful to enhance the detection of the suspicious regions in the MRI. Accessing and researching real medical pictures akin to those of MRI, PET or CT scan could be a terribly advanced task as a result of confidentiality problems as well as significant methodological hurdles.

Nowadays, tomography systems square measure vital in medical image process and analysis.

The sample MRI Images are as shown below in figure 1. Depending upon the relaxation times of the fields applied while capturing the MRI of the patients, there are three types T1 weighted, T2 weighted and Flair. 


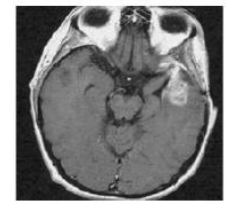

A

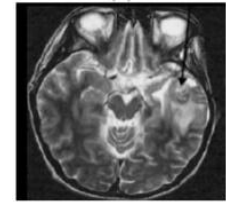

B

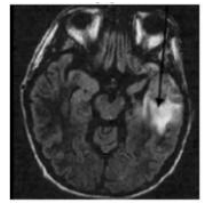

$\mathrm{C}$

Figure. 1 A) T1-Weighted B) T2- Weighted C) Flair

According to radiologic appearance of Tumours they can be categorized as mentioned in the Table 1 . The appearance of the tumor is also differing in $\mathrm{T} 1$ and $\mathrm{T} 2$ weighted images

Table 1. Classification of tumors

\begin{tabular}{|l|l|l|}
\hline Type of Tumor & Type of MRI & Appearance \\
\hline \multirow{2}{*}{ Non-enhanced } & T1 weighted & Hypointense \\
\cline { 2 - 3 } & T2 weighted \& Flair & Hyperintense \\
\hline \multirow{2}{*}{ Full-enhanced } & T1 weighted & Hypointense \\
\cline { 2 - 3 } Full enhanced with edema & T2 weighted \& Flair & Hyperintense \\
\hline \multirow{2}{*}{ Ring enhanced tumor } & \multirow{2}{*}{ Same as above } & \\
\hline
\end{tabular}

Three Major steps in Patient treatment from research point of view are

1. To examine and verify that a structural abnormality is there or not there.

2. Further to locate and evaluate the extent of any abnormality.

3. To exemplify the irregularity if present.

To perform the above-mentioned steps in Brain Tumor Detection, three basic steps are as shown in figure 2.

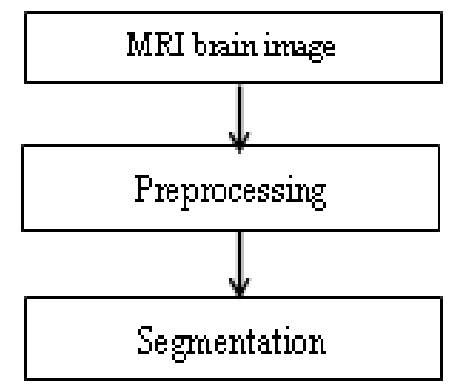

Figure 2. Basic step ladder for MRI processing

To perform these steps many researches have used different techniques. different methods are being used by the researchers for performing tumor identification previously. Some are listed in Table 2.

Table 2. State of art methods of MRI Processing

\begin{tabular}{|l|l|l|}
\hline Preprocessing & Enhancement & $\begin{array}{l}\text { Feature extraction and } \\
\text { Segmentation }\end{array}$ \\
\hline Fourier transform techniques & Prewitt edge-finding filter & Seeded Region Growing \\
\hline Standard imaging protocol & Median filter [16] & Markov Random Field model \\
\hline $\begin{array}{l}\text { Histogram based algorithms [11] } \\
{[16][17]}\end{array}$ & Low pass Filter & Active contour model \\
\hline Pixel Histograms [11] [16] & Triple Quantum Filtered & Fuzzy methods C-mean Algorithms \\
\hline Content based models & Edge Finding filter, & K-mean clustering Algorithms \\
\hline
\end{tabular}


PEN Vol. 7, No. 3, September 2019, pp.1102- 1113

\begin{tabular}{|l|l|l|}
\hline Geometric Prior, Primodel & Novel image Approach & $\begin{array}{l}\text { Texture Features [9] [10] [11] } \\
\text { self-organizing map }\end{array}$ \\
\hline $\begin{array}{l}\text { Independent component analysis } \\
\text { (ICA) }\end{array}$ & Prewitt edge-finding filter & Adaptive Histogram Analysis \\
\hline $\begin{array}{l}\text { Principal component analysis } \\
\text { PCA) }\end{array}$ & Genetic Algorithm & Automatic 2D segmentation \\
\hline $\begin{array}{l}\text { Unseeded region growing } \\
\text { algorithm }\end{array}$ & Gabor Filter [12] & Support vector machine \\
\hline Neural networks [9] [17] & Morphological Filter & Genetic Algorithm \\
\hline Statistical parametric Mapping & Gaussian Filter domain & AdaBoost [11] \\
\hline DWT [10] & $\begin{array}{l}\text { Frequency } \\
\text { enhancement }\end{array}$ & $\begin{array}{l}\text { 1ST order features, GLCM, } \\
\text { GLRLM, } \\
\text { [12] }\end{array}$ \\
\hline Mutual Information & & \\
\hline
\end{tabular}

From the literature survey it has been observed that following challenges exists in the Brain tumor Detection:

1. Precise and reproducible segmentation \& Characterization of abnormalities are still a demanding and difficult task

2. Still there is significant room for getting increased automation, applicability and accuracy in the existing methods.

The major Difficulty faced by the researchers in MRI processing is the diversity of the feasible shape, location and picture intensities of a range of nature of tumours.

Most of the medical imaging studies and detection square measures included antilepton emission imaging (PET) and computerized axial tomography (CT) scanning. Tomography features a dimensional nature and provides completely different information $[4,5]$.

The remaining paper is structured like follow; section two demonstrates the brain symmetry and its clinical implications. Section three included the brain midsagittal plane detection techniques developed by researchers. Section four describes the proposed approach of the brain hemisphere separation and also illustrates the simulation results. Section V included conclusion and future of the work.

\section{Background}

Bilateral symmetry is an important aspect of the biology in the humans. Our outlook exhibits symmetricalness in the organs of our body and very few are available in the symmetrical pair off. The neural structure of the individual brain is split into two hemispheres, split by the inter-hemispheric fissure (IF). Assessments of the two hemispheres and finding of variations have been a subject of interest for several years. Traditional human brains exhibit associate fairly accurate symmetricalness with reference to the inter-hemispheric (longitudinal) fissure bisecting the brain, referred to as the anatomical midsagittal plane (MSP). The right and left lobe structures are similar in nature. Notwithstanding, the two halves of the hemispheres have around indistinguishable anatomical properties. Radiologists routinely utilize symmetry as a standout amongst the most separating highlights in conjunction with different characters, for example, area, neighbor-hood relationship, and shape, to survey variations from the norm in brain pictures. An expert clinician identifies the symmetry axis/plane by looking at a medical image and comparison of the abnormality is done with the healthy side of the brain [7]. Nonetheless, human brains are never flawlessly symmetric. Obsessive brains, specifically, frequently leave radically from reciprocal symmetry. For successful neurotic brain picture arrangement and examination, it is most important to characterize a plane of location that is invariant for the symmetrical and also topsy-turvy cerebrum pictures, and it is also important to create calculations that catch this reference plane vigorously [1] 
Table 3. Existing methods for symmetry planes detection

\begin{tabular}{|c|c|c|c|}
\hline Methods & Take out feature & $\begin{array}{l}\text { limited search vs. universal } \\
\text { search }\end{array}$ & Capturing Equipments \\
\hline Brummer (1991) & IF & $\begin{array}{l}\text { universal: seek longitudinal } \\
\text { fissure }\end{array}$ & MRI \\
\hline $\begin{array}{l}\text { Hu and Nowinnski } \\
\text { (2003) }\end{array}$ & IF & partial: in the vicinity of IF & MRI, CT \\
\hline Minovic (1993) & Main axes & $\begin{array}{l}\text { universal: search the inertia } \\
\text { matrix of } 3 D \text { rigid body }\end{array}$ & $\begin{array}{l}\text { Simulated data and } \\
\text { MRI }\end{array}$ \\
\hline $\begin{array}{l}\text { Liu } \\
(2006)\end{array}$ & outside plane tip cloud & universal & $\begin{array}{l}\text { Simulated data and } \\
\text { MRI }\end{array}$ \\
\hline $\begin{array}{l}\text { Liu } \\
(2001)\end{array}$ & $\begin{array}{l}\text { border map cross } \\
\text { relationship }\end{array}$ & $\begin{array}{l}\text { universal: edge cross } \\
\text { correlation }\end{array}$ & MRI, CT \\
\hline $\begin{array}{l}\text { Smith and Jenkinson } \\
\text { (1999) }\end{array}$ & $\begin{array}{l}\text { The ratio of intensity } \\
\text { profile }\end{array}$ & $\begin{array}{l}\text { universal: measure the } \\
\text { symmetry of the lines } \\
\text { orthogonal to the candidate } \\
\text { symmetry plane }\end{array}$ & $\begin{array}{l}\text { CT, MRI, PET, } \\
\text { SPECT }\end{array}$ \\
\hline Junck (1990) & $\begin{array}{l}\text { contented cross } \\
\text { relationship }\end{array}$ & universal & $\begin{array}{l}\text { PET, } \\
\text { SPECT }\end{array}$ \\
\hline Ardekani (1997) & $\begin{array}{l}\text { contented cross } \\
\text { relationship }\end{array}$ & partial: on a unit circle & MRI, PET \\
\hline Prima (2002) & $\begin{array}{l}\text { contented cross } \\
\text { relationship }\end{array}$ & partial: block matching & CT, MRI, PET, SPECT \\
\hline Volkau (2006) & $\begin{array}{l}\text { concentration } \\
\text { distribution } \\
\text { Kullback-Leibler' } \\
\text { measure }\end{array}$ & universal & MRI, MRA, CT \\
\hline
\end{tabular}

\section{Research in brain symmetry determination}

The highly symmetrical distribution provides an excellent opportunity for researcher to develop symmetryaided diagnosis techniques. Many researchers are motivated and have been developing many symmetry integrated methods for brain image analysis. In order to retrieve symmetry information from a brain image, it is crucial to identify the precise location of the brain symmetrical plane. Various strategies have been proposed for the brain symmetry location [7].

In radiological scanning process, the brain images obtained may be disoriented. The examined cerebrum pictures are fairly tilted and misshaped. Tilt and contortion can prompt visual wrong review and frequently yield false clinical elucidation, since cuts of the mind pictures are never concordant with the homologous 
structures inside a similar coronal or hub level. The incline of the head is regularlyunderstood in the toolamong the examinationtechnique, nevertheless, is not generally manageable.

Regular tenacities behind these sorts of tilts may incorporate; however, are not restricted to fixed status of patients, the freshness of the experts and imprecision of alignment frameworks.

Correcting the incline of the head is relational to rearranging the midsagittal plane (MSP) with the principal point of the image cross-section. The MSP is categorized as the level that finestseparates the brain into two portions. It is apparent that the rearrangement of the MSP from the geometrical deception yields more sensible information evaluation either by a human master or a PC program that depends on side of the equator insightful cross-referencing.

Symmetry axis is considered as the best isolating central planar brain pictureinto two parts in 2-D applications. For identifying, dichotomizing, calculating and applying symmetry in imageexaminationthere have been many methodologies proposed in the literature. The representative route for deciding symmetry chopperexpect the protest is to approximatelygradelengthy so the banner of the hub of least inactivity can be utilized to characterize the symmetry chopperof a 2D planar shape. This is the pivot about which the second snapshot of a thin sheet of material of a similar shape is the littlest. The subtle elements of existing strategies for identifying symmetry planes of cerebrum pictures are classified beneath. It ought to be noticed that for any of these calculations, the Resilience to typical asymmetries is the basic of the achievement of anomalous asymmetry recognition. An essential point is that the asymmetry examination as an apparatus for pathology location can't be accomplished without separating ordinary asymmetries from irregular asymmetries [4].

The major challenges to be considered while symmetry analysis can be:

(1) Regular asymmetry in the image signal intensity,

(2) Irregular asymmetry in the image signal intensity, e.g. the nearness of tumor.

(3) Unwanted signals due to geometrical misalignment;

(4) Unwanted signals due to non-homogeneity of the indications, e.g., inclination fields.

The calculation shows an edge-based, cross-connection approach that breaks down the plane fitting issue into revelation of two- dimensional symmetry chopperon each cut, trailed by a vigorous estimation of plane parameters. The calculation's resilience to cerebrum asymmetries, input picture balances and picture commotion is quantitatively assessed. The outcomes noted that the calculation can separate the iMSP from input 3-D pictures with extensive topsy-turvy sores, subjective starting revolution balances, low flag tocommotion proportion or high predisposition field. The iMSP calculation is contrasted and an approach in light of augmentation of shared data enlistment, and is found to show unrivaled execution under antagonistic conditions. At long last, it is likewise observed that no factually critical distinction is found between the midsagittal plane processed by the iMSP calculation that assessed by two prepared neuroradiologists. [1] A mechanized PC helped with perfect midline estimation framework with two-stages incorporates CT Slice Selection Algorithm (SSA) and Ideal midline identification (IML location) involved in the center of the framework. SSA is utilized to extraordinarily lessen the cut number while the IML discovery is intended to precisely distinguish the IML position and turn edge.

This database available includes 3133 pivot CT scan slices. The images have been acquired crosswise over 70 patients with cases including both gentle and serious Traumatic Brain Injuries (TBI). All the accessible CT examinations have been used in testing the framework's procedures and in the estimation of the perfect midline.

A thorough symmetric position seek is performed in light of the anatomical elements in the discovery. Remembering thefinishtarget to promote the precision of the recognition, a worldwide revolution suspicion is connected to decide the perfect midline by completely considering the association between cuts. Trial consequences of the multi-organize calculation were evaluated on $3313 \mathrm{CT}$ cuts of 70 patients. The exactness of the proposed framework is $96.9 \%$. To use any method for the clinical settings exactness is important. So, this feature makes it practical for use under clinical settings [2]. 
Pixel esteems-based symmetry investigation comprises of three stages. In the primary stage, DICOM records input are changed over into required RGB and Gray arrangements. In the second stage symmetry remainder exhibit in the Axial human cerebrum MRI is figured with the assistance of another parameter as mean pixel esteem angle. X-ray is apportioned into standard size dim level picture into eight constituent modules. The obtained gray level values are observed with more difference in case of asymmetry and likely to be same with some tolerance for normal cases. The method fails to detect the abnormality located on the center line, in which case the symmetry property is retained. [8]

\section{Proposed algorithm and simulation results}

The proposed algorithm reads the image from the database. Pre-processing of the image is done using median filter. Then, it divides the 2D MRI images into two halves, i.e. left and right, based on the mutual information present in the image.

Based on the mutual information it is decided which half part to process further. First order and second order statistical features are extracted to authenticate the tumor presence or absence.

Similarly, the algorithm is developed to divide the pre-processed image into six and eight equal parts. Extraction of statistical features of complete image is not ending with the optimum results. Hence to improve the algorithm efficiency, number of subsections is done and then features are extracted. The images used for the experimentation are T2 weighted images. The algorithm is applied on the around $30 \mathrm{MRI}$ images available in the database. [10]

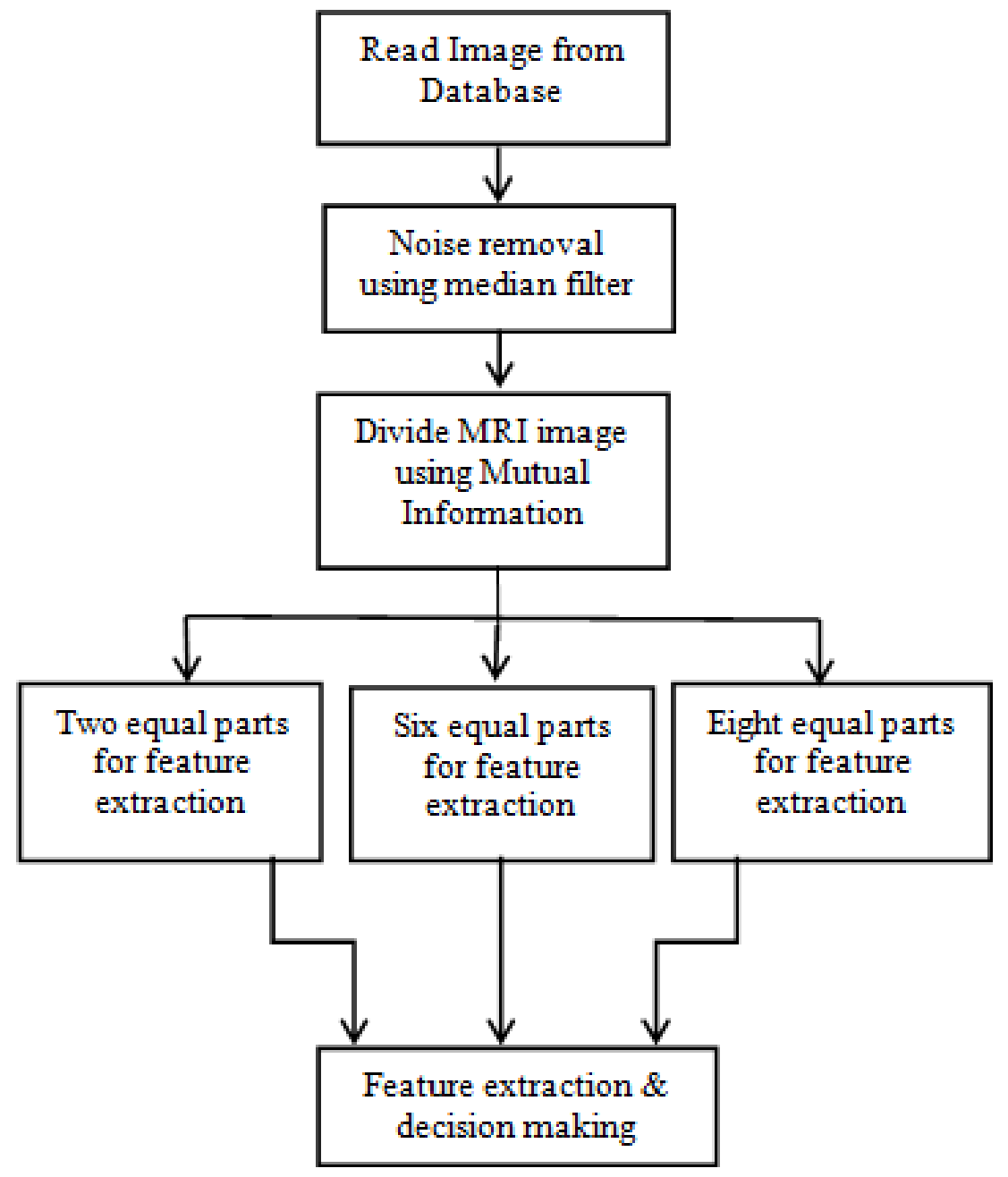

Figure.3 Proposed algorithm 

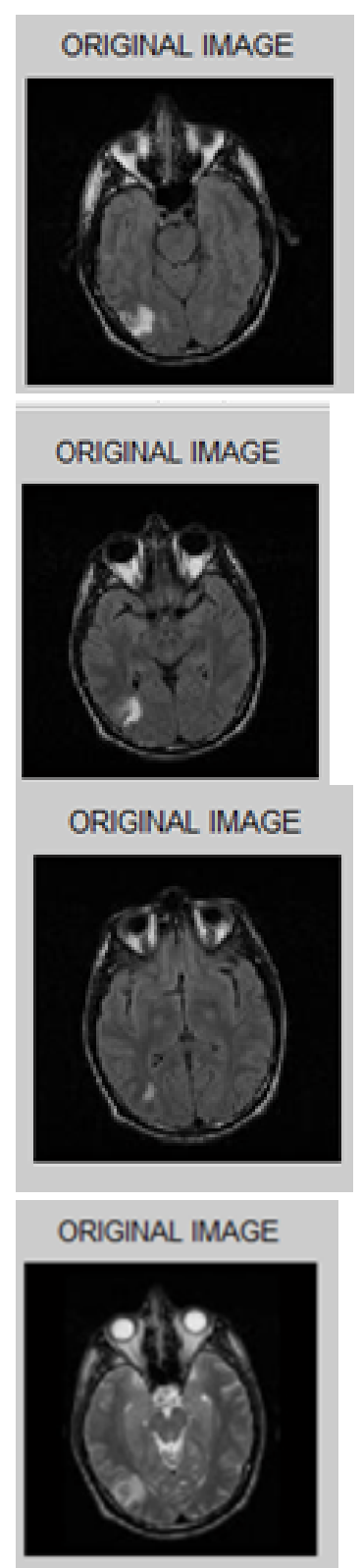

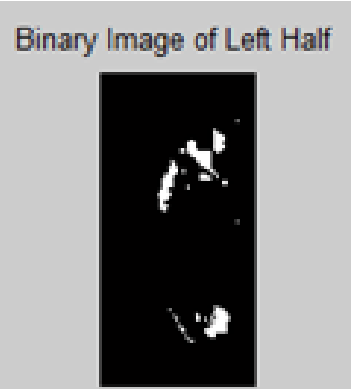

Binary Image of Left Half

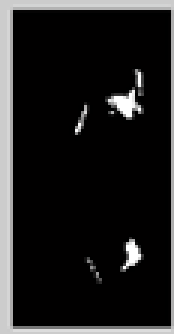

Binary Image of Left Half

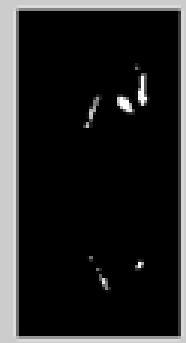

Binary Image of Left Half

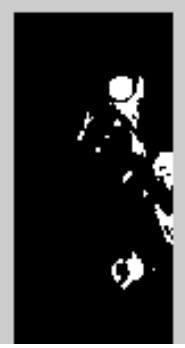

Binary Image of Right Half

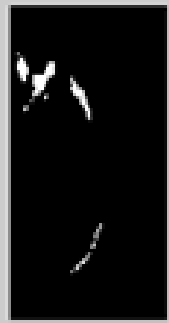

Binary Image of Right Half

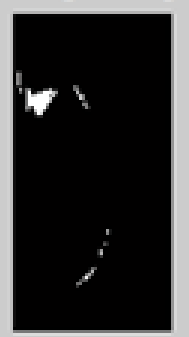

Binary Image of Right Half

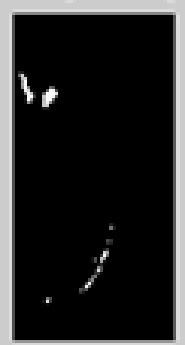

Binary Image of Right Half

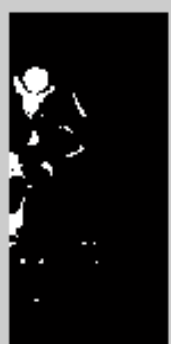

Figure 4. Output of proposed algorithm with axial image divided into two equal parts

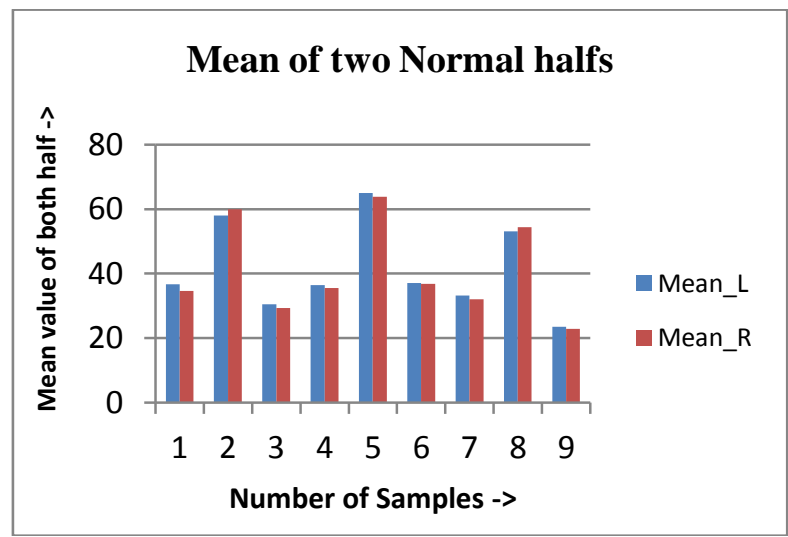

Figure 5. Graph of mean of two halves without tumor 


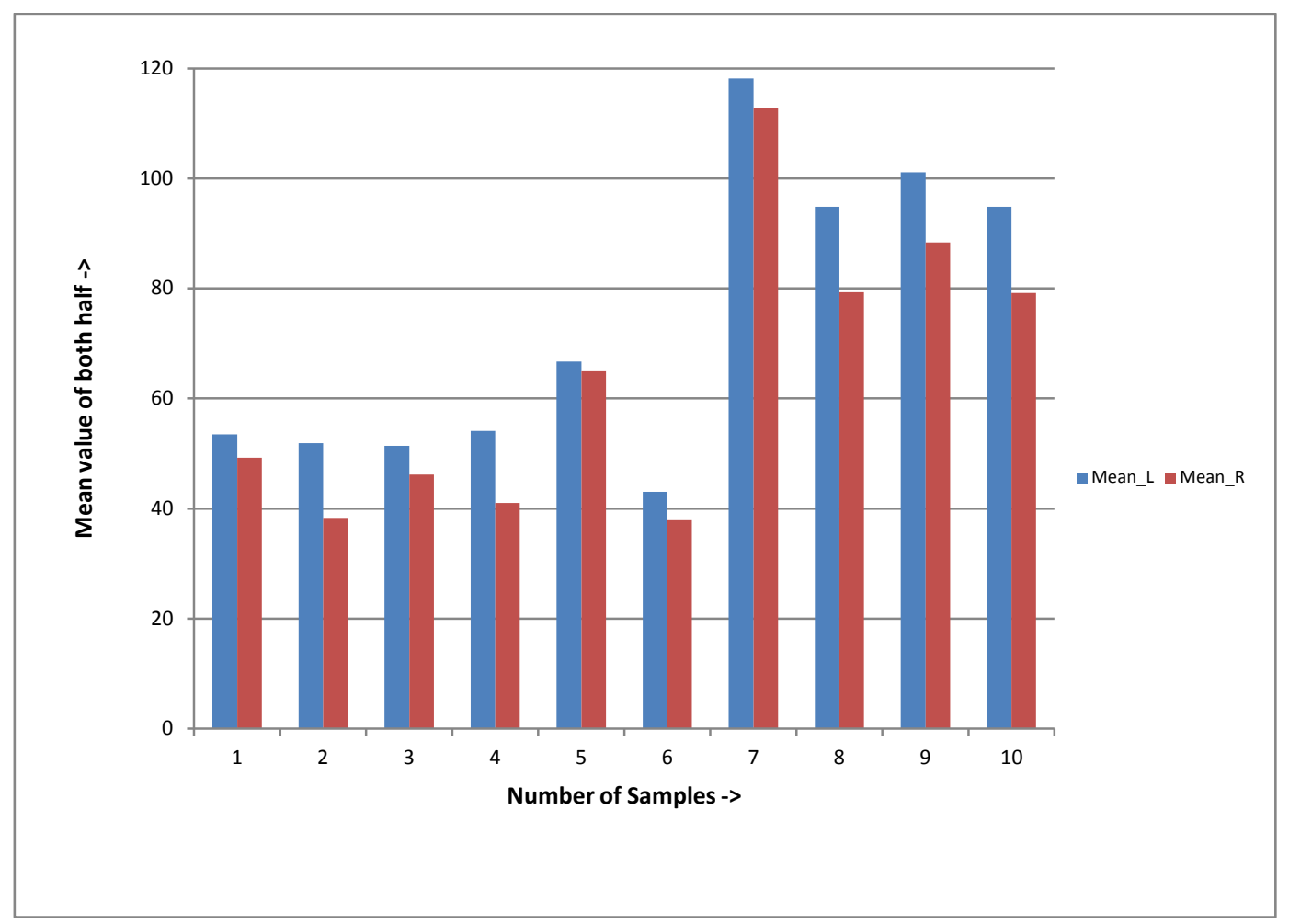

Figure 6. Graph of mean plotted when tumor is in left half

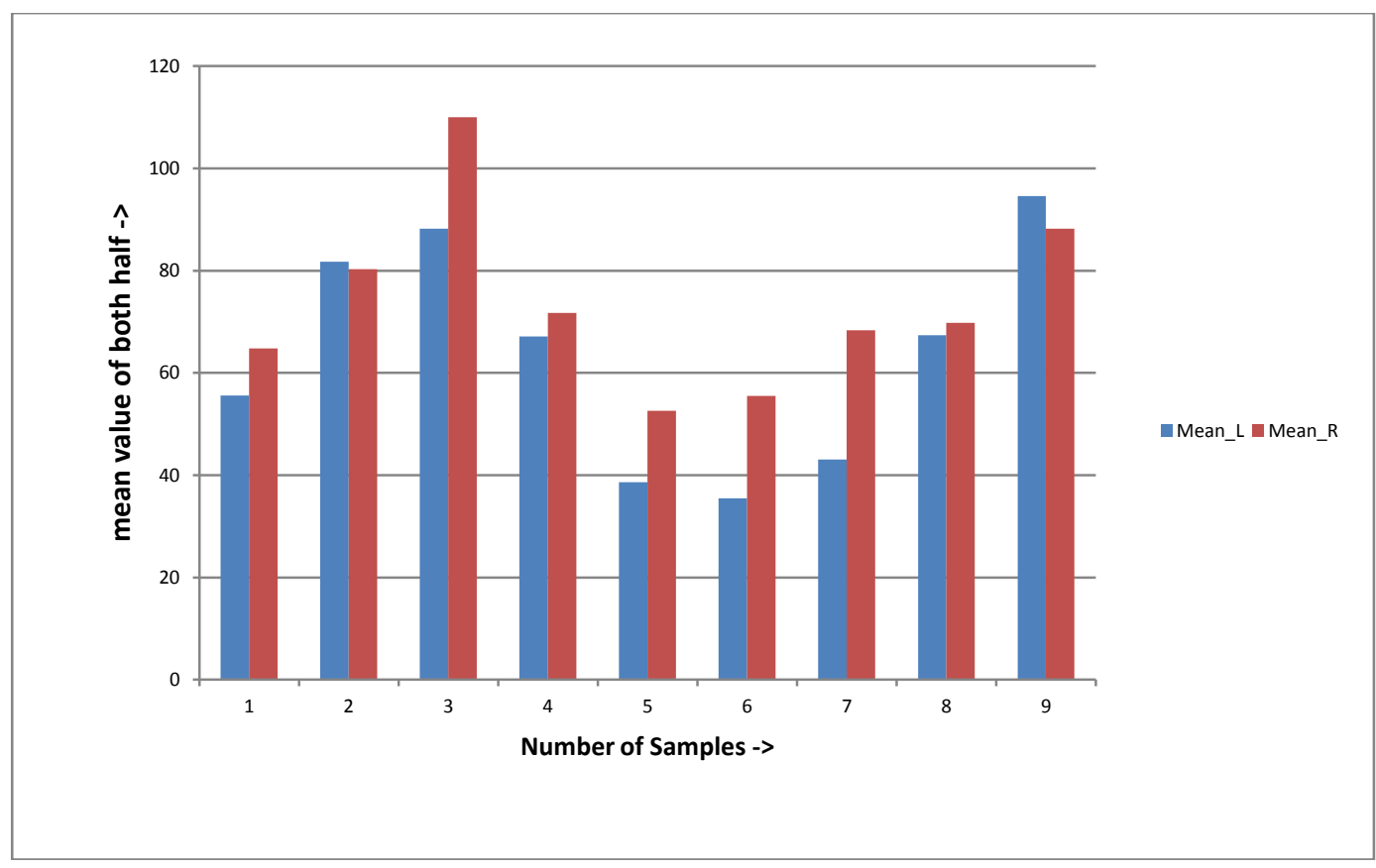

Figure 7. Graph of mean plotted when tumor is in right half

From the obtained graphs as shown in above in figure 5, 6 and 7 it has been observed that there is negligible difference in the mean value of left and right half in case of chart plotted for MRI scans of normal patient. When tumor is present in the righthalf part rise in mean value is observed for left part and similar observations are seen for images with tumor in the right half portion which is also reflected in the charts plotted. 

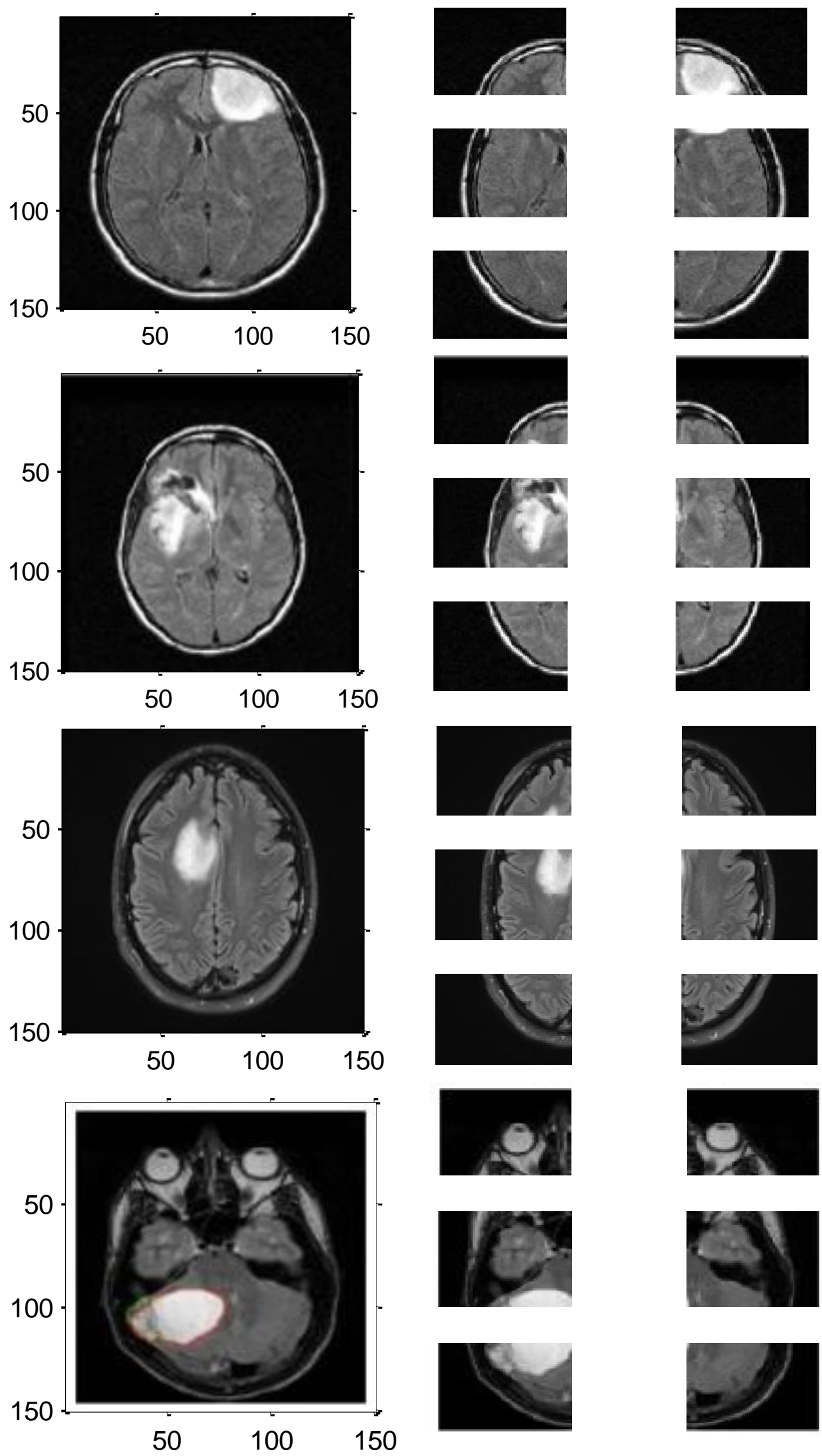

Figure 8. Output of proposed algorithm with axial images divided into two six equal parts 

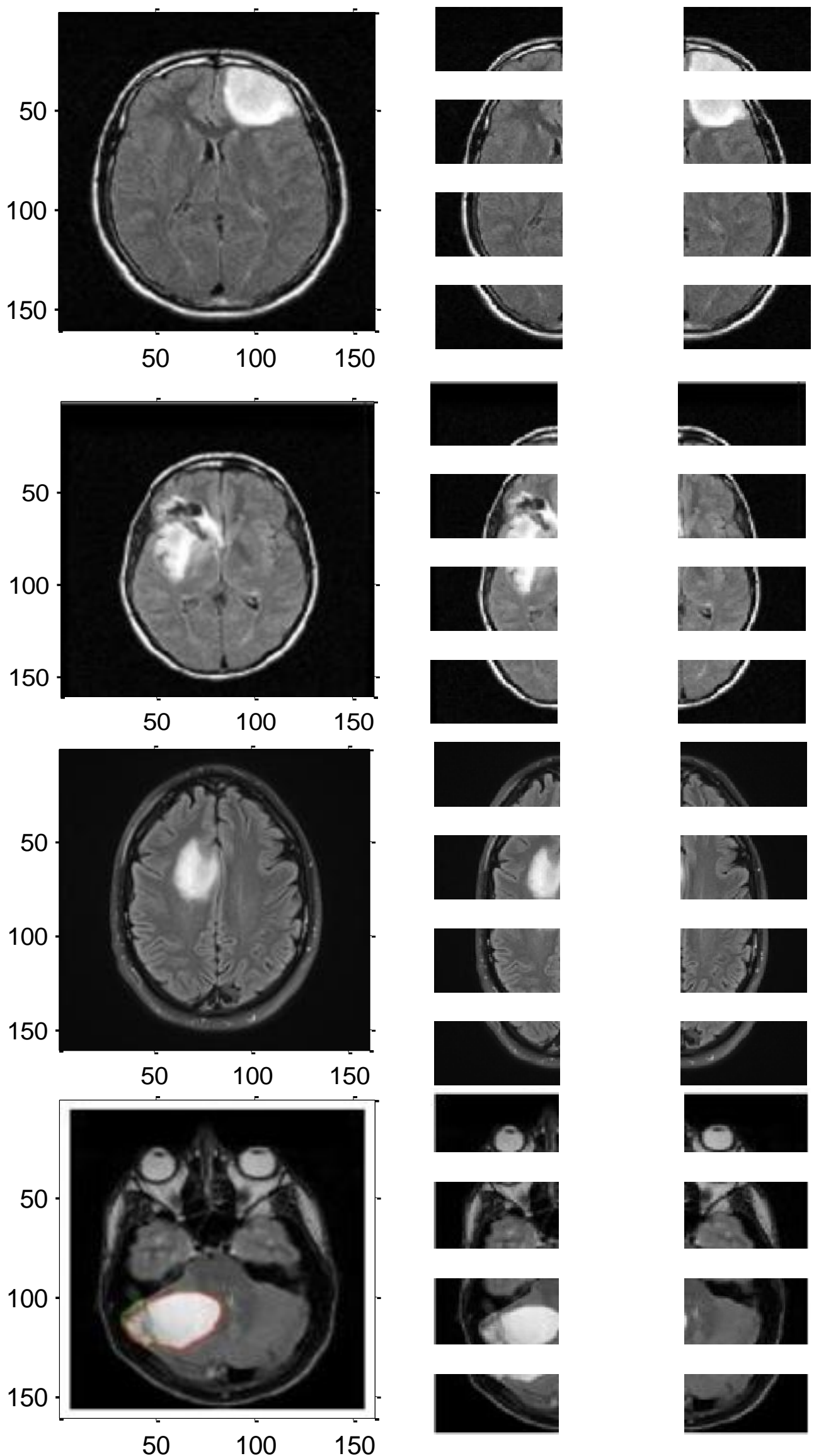

Figure 9. Output of proposed algorithm with axial images divided into two eight equal parts 


\section{Conclusion and future scope}

There are many algorithms in the literature working on the principal of symmetry property of brain. The first part of proposed algorithm pre-processes the image to remove artefacts, to correct the orientation if any and filtration etc. Then, divides the 2D MRI images into two halves i.e. left and right. It also considers the tilts occurred during the capturing of MRI images. Further, the decision is done on which half to process further. The statistical first order, second order features can be extracted from selected half. Also, the work is extended to divide the image instead of two into either six or eight parts. The decision of detecting tumor present or not is taken based on the features extracted. It is observed that when the image is divided into six or eight it becomes prominent to detect the presence of the tumor.

The extracted the features of brain MRI improve the detection rate of tumor when number of sections is increased. In future, the algorithm can be extended to 3D application to calculate the volume. Also, the algorithm can be extended to the problem were the tumor is lying exactly at the center i.e. on the mid line and symmetrically grown on both the sides.

capitalize only the first word in a paper title, except for proper nouns and element symbols. If you are short of space, you may omit paper titles. However, paper titles are helpful to your readers and are strongly recommended. For papers published in translation journals, please give the English citation first, followed by the original foreign-language citation [8].

\section{References}

[1] Yanxi Liu, Robert T. Collins, and William E. Rothfus, "Robust Midsagittal Plane Extraction from Normal and Pathological 3-D Neuroradiology Images,", IEEE Transactions On Medical Imaging, VOL. 20, NO. 3, pp 175 - 192, MARCH 2001

[2] Xuguang Qi, Ashwin Belle, Sharad Shandilya, Wenan Chen, Charles Cockrell, Yang Tang, Kevin R. Ward, Rosalyn H. Hargraves, Kayvan Najarian, "Ideal Midline Detection Using Automated Processing of Brain CT Image", Open Journal of Medical Imaging, pp 51-59, 2013.

[3] Dvorak, P, Brno, Czech Republic ; Kropatsch, W. ; Bartusek, K., "Automatic detection of brain tumors in MR images" Telecommunications and Signal Processing (TSP), 36th International Conference., pp 577 580, 2-4 July 2013

[4] Sheena Xin Liu, "Symmetry and asymmetry analysis and its implications to computer-aided diagnosis: A review of the literature", Journal of Biomedical Informatics, pp 1056-1064, 2009.

[5] Dong-Hyun Kim and Soo-Young Ye, "CAD for Detection of Brain Tumor Using the Symmetry Contribution From MR Image Applying Unsharp Mask", Transactions On Electrical And Electronic Materials, Vol. 15, No. 4, pp. 230-234, August 25, 2014

[6] H.J. Kuijf, S.J. van Veluw, M.I. Geerlings, M.A. Viergever, G.J. Biessels, K.L. Vincken, "Automatic extraction of the midsagittal surface from brain MR images using the Kullback Leibler measure", Neuroinformatics, nr. 3, vol. 12, pp. 395-403, 2014.

[7] Surani Anuradha JayasuriyaAlan Wee-Chung Liew, Phillip Sheridan, "Symmetry Detection in Brain Image Analysis", IGI Global/ Medical Technologies, pp 5615-5617, 2015.

[8] Kirti Raj Bhatele, Sarita Singh Bhadauria, "Pixel based Symmetry Analysis of an Axial T2 Weighted Brain MRI", IJCA, Volume 118 - No.24, pp 9-14, May 2015

[9] Neeraj Sharma, Amit K. Ray, Shiru Sharma, K. K. Shukla, SatyajitPradhan, and Lalit M. Agarwal, "Segmentation and classification of medical images using texture-primitive features: Application of BAM-type artificial neural network", J Med Phys. Jul-Sep; 33(3): 119-126, 2008.

[10] Namita Aggarwal, R. K. Agrawal, "First and Second Order Statistics Features for Classification of Magnetic Resonance Brain Images", Journal of Signal and Information Processing, pp.146-153, 2012

[11]Xiao Xuan, Qingmin Liao, "Statistical Structure Analysis in MRI Brain Tumor Segmentation", ICIG[Fourth International Conference on Image and Graphics], pp 421 - 426, 2007 IEEE.

[12] Nooshin Nabizadeh, Miroslav Kubat, "Brain tumors detection and segmentation in MR images: Gabor wavelet vs. statistical features", Elsevier, Computers and Electrical Engineering, pp 1 - 16, 2015.

[13] Mussarat Yasmin, Sajjad Mohsin, Muhammad Sharif, MudassarRaza and Saleha Masood,"Brain Image Analysis: A Survey", World Applied Sciences Journal 19 (10), ISSN 1818-4952: 1484-1494, 2012

[14] R K Samantaray1, S B Panda, B Pradhan, "Automated Brain Tumor Detection and Identification Using Image Processing”, Researcher, http://www.sciencepub.net/researcher, pp 79-88, 2013. 
[15]Rash Bihari Dubey, Madasu Hanmandlu, Suresh K. Gupta, Sushil K. Gupta, "The Brain MR Image Segmentation Techniques and use of Diagnostic Packages", Academic Radiology, Vol 17, No 5, pp 658 671, May 2010.

[16] Natarajan P, Krishnan. N, Natasha Sandeep Kenkre, Shraiya Nancy, Bhuvanesh Pratap Singh, "Tumor Detection using threshold operation in MRI Brain Images ”, International Conference on Computational Intelligence and Computing Research, pp. 1-4, 2012 IEEE

[17]Jasmin Jasko Sutkovic "A review on Nanoparticle and Protein interaction in biomedical applications" Periodicals of Engineering and Natural Sciences Vol. 4 No. 2 (2016), pp. 1 - 10

[18] Pavel Dvorak, Walter Kropatsch, and KarelBartusek, "Automatic Detection of Brain Tumors in MR Images", IEEE TSP, pp. - 577 - 580, 2013

Osman Gursoy, Md. Haidar Sharif "Parallel Computing for Artificial Neural Network Training", Periodicals of Engineering and Natural Sciences Vol. 6, No. 1, (2018), pp. $1-10$ 